\title{
BOOKS
}

\section{All-inclusive text on candidiasis} Candidiasis: Pathogenesis, Diagnosis and Treatment, 2nd edn, edited by Gerald P Bodey (1993). Raven Press, Ltd, 1185 Avenue of the Americas, New York, New York 10036, USA. 432 pages, US $\$ 95.00$

Infections caused by Candida species have emerged as a prominent entity in the latter part of the 20th century. This trend is exemplified by candidemia, which was considered to be rare 40 years ago, but today assumes a significant role as a nosocomial infection. The increasing role of both superficial and systemic candida infections is directly related to the mushrooming population of immunocompromised patients with cancer, organ transplantation, AIDS and on long term immunosuppressive agents.

Writing a textbook on the pathogenesis, diagnosis and treatment of candidiasis is a daunting task. In his second edition on this subject, Dr Gerald Bodey has assembled an array of prominent experts in their respective fields. The compilation of their work is a book that is generally well written and full of pertinent information about candidiasis. The editor has divided the book into sections on the microbiology of Candida species, pathological correlations, epidemiology of candidiasis, laboratory diagnosis, radiological features, clinical manifestations of candida syndromes and, finally, therapeutic options. There are sections of the text, however, that are poorly organized and that present data superficially.

Worthy of praise are the chapters Biology and Pathogenicity, Pathogenesis, Epidemiology, Laboratory Diagnosis, Oral Candidiasis, Genital Candidiasis, Neonatal Candidiasis, Hematogenous and Organ Candidiasis, Candida Ophthalmitis and Central Nervous System Infection, and Antifungal Agents that summarize the state of the art of these subjects. The inclusion of data on candida hypersensitivity syndrome exemplifies the all-inclusive nature of this book.
However, the chapters describing pathological correlations and animal models are superficial and lack the insight one would expect in this type of book. The presentation of the radiological features of candida infections is poorly organized. It is formulated along a radiographic organ system approach rather than according to clinical syndromes. The lateral approach may have proven more useful to clinicians. The authors failed to detail the advantages of one radiographic procedure compared with another in the diagnosis of candidiasis. The use of sensitivity and specificity measures would have been beneficial for the reader in deciding which investigative procedure is warranted. The authors' report on cutaneous candidiasis is also superficial. Particularly deficient is the treatment of cutaneous candidiasis, although this topic is covered in more detail in a later chapter on antifungal agents. In addition, the chapter on urinary candidiasis should have been expanded to include the current thought on candiduria in intensive care unit patients and the predisposition of these patients to systemic candidiasis. Some thoughts on colonization with Candida species in the intensive care unit setting and the concomitant effect of this colonization on mortality would have been welcome. The inclusion of prophylactic antifungal trials currently underway to prevent candida colonization in this clinical setting would have been prudent. Finally, on more than two occasions, the content of one chapter overlapped with that of another.

Despite its flaws, the second edition of Candidiasis: Pathogenesis, Diagnosis and Treatment offers a comprehensive look at this emerging subject. It is a worthwhile addition to one's library and serves as a jumping off point for the clinician desiring to probe further into the ever expanding field of clinical mycology.

Coleman Rotstein MD Henderson General Hospital - McMaster Medical Unit Hamilton, Ontario 


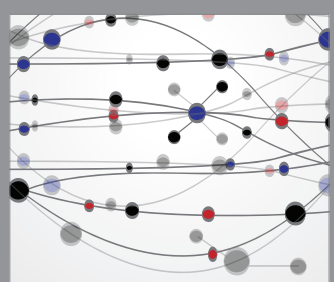

The Scientific World Journal
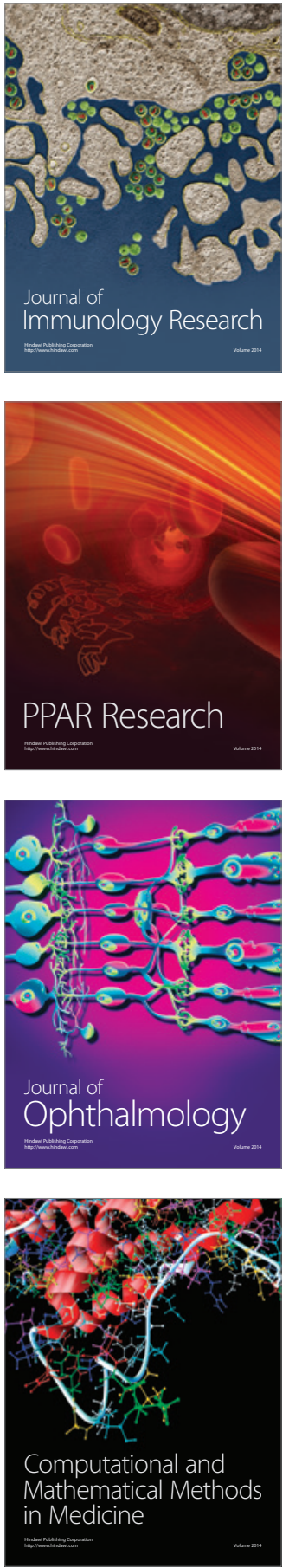

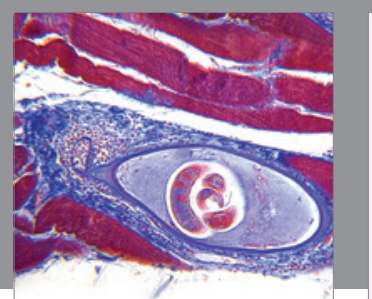

Gastroenterology Research and Practice

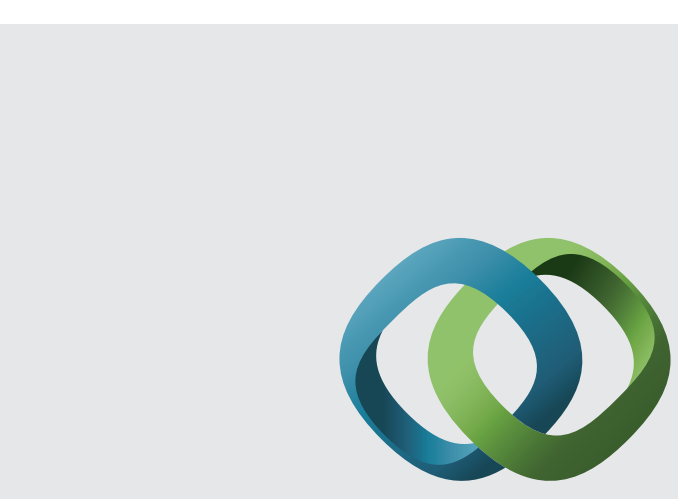

\section{Hindawi}

Submit your manuscripts at

http://www.hindawi.com
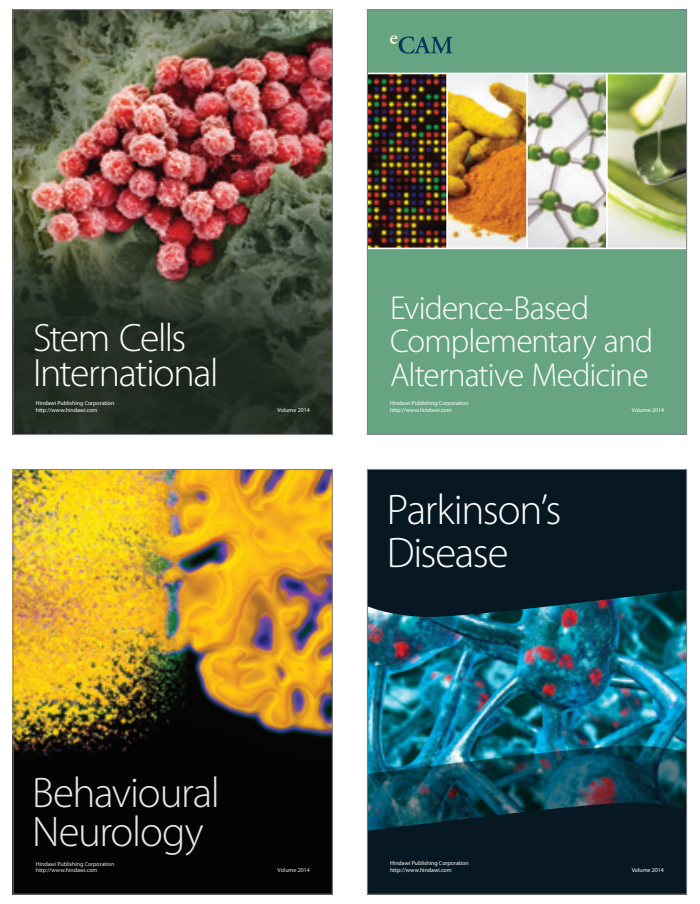
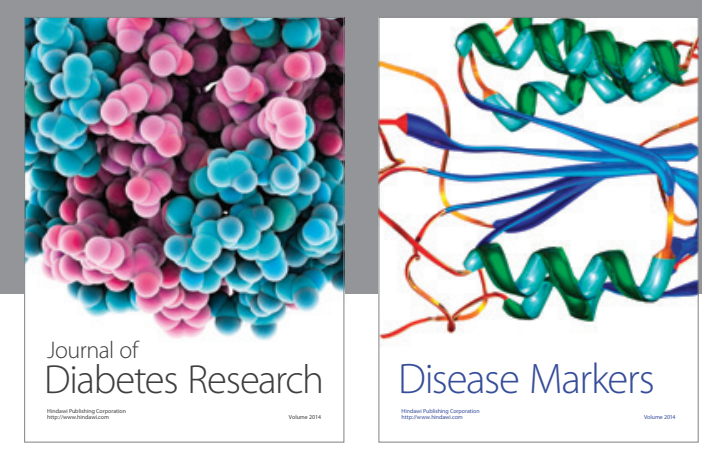

Disease Markers
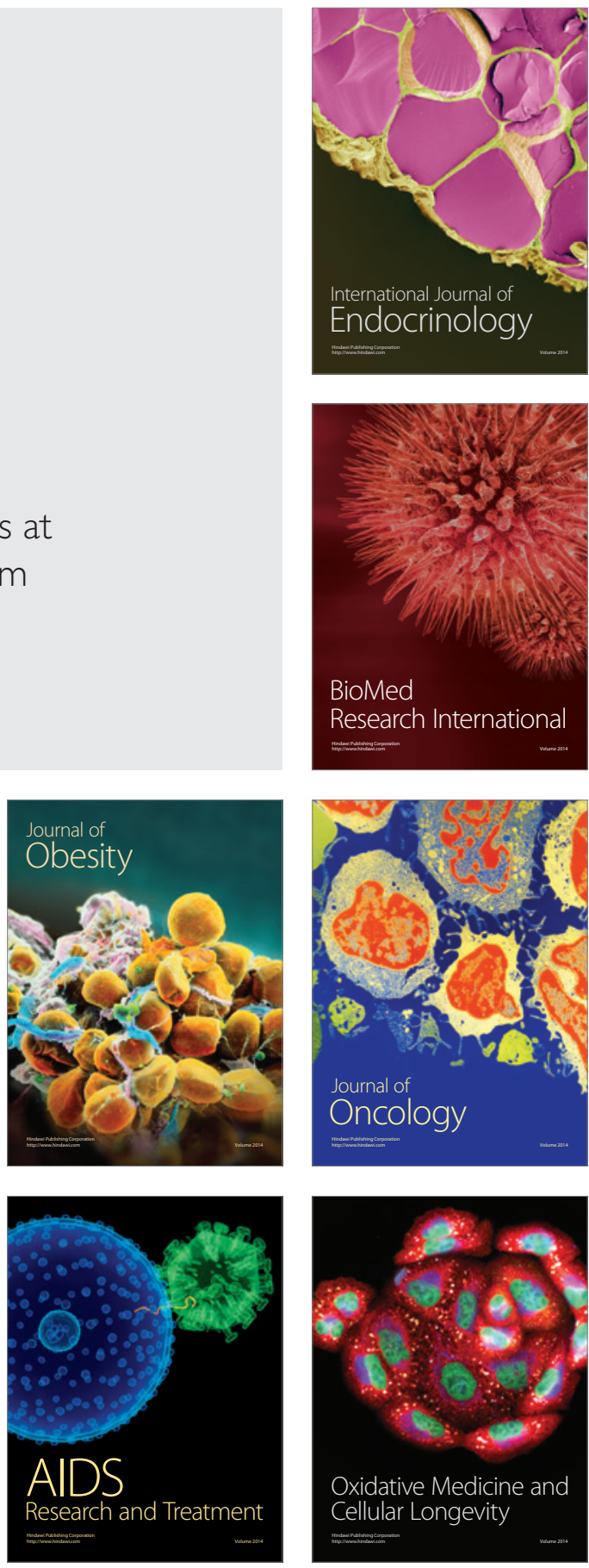